\title{
Optimization and Performance Study of a Proton CT System for Pre-Clinical Small Animal Imaging
}

\author{
Sebastian Meyer ${ }^{1, *}$, Jonathan Bortfeldt ${ }^{1,2}$, Paulina Lämmer ${ }^{1}$, \\ Franz S. Englbrecht ${ }^{1}$, Marco Pinto ${ }^{1}$, Katrin Schnürle ${ }^{1}$, \\ Matthias Würl ${ }^{1}$ and Katia Parodi ${ }^{1}$ \\ ${ }^{1}$ Department of Medical Physics, Faculty of Physics, Ludwig-Maximilians-Universität \\ München, Garching b. München, Germany \\ ${ }^{2}$ CERN, Geneva, Switzerland \\ *Current affiliation: Department of Radiology, Perelman School of Medicine, \\ University of Pennsylvania, Philadelphia, USA \\ E-mail: Sebastian.Meyer@pennmedicine.upenn.edu
}

29 May 2020

\begin{abstract}
Proton computed tomography (pCT) promises to reduce or even eliminate range uncertainties inherent in the conversion of Hounsfield units into relative stopping power (RSP) for proton therapy treatment planning. This is of particular interest for proton irradiation studies in animal models due to the high precision required and uncertainties in tissue properties.

We propose a dedicated single-particle tracking pCT system consisting of low material budget floating strips Micromegas detectors for tracking and a segmented time-projection-chamber with vertical Mylar absorbers, functioning as a range telescope. Based on Monte Carlo simulations of a realistic in silico beam and detector implementation, a geometrical optimization of the system components was conducted to safeguard an ideal operation close to intrinsic performance limits at $75 \mathrm{MeV}$. Moreover, the overall imaging capabilities relevant for pre-clinical proton therapy treatment planning were evaluated for a mouse model.

In order to minimize extrinsic uncertainties in the estimated proton trajectories, a spacing of the two tracking planes of at least $7 \mathrm{~cm}$ is required in both tracking detectors. Additionally, novel in-house developed and produced aluminum-based readout electrodes promise superior performance with around $3 \mathrm{~mm}^{-1}$ spatial resolution due to the reduced material budget. Concerning the range telescope, an absorber thickness within $500 \mu \mathrm{m}$ to $750 \mu \mathrm{m}$ was found to yield the best compromise between waterequivalent path length resolution and complexity of the detector instrumentation, still providing sub-0.5\% RSP accuracy. The optimized detector configuration enables better than $2 \%$ range accuracy for proton therapy treatment planning in pre-clinical data sets.

This work outlines the potential of pCT for small animal imaging. The performance of the proposed and optimized system provides superior treatment planning accuracy compared to conventional X-ray CT. Thus, pCT can play an important role in translational and pre-clinical cancer research.
\end{abstract}

This is an author-created, un-copyedited version of an article accepted for publication 
in Physics in Medicine \& Biology. IOP Publishing Ltd is not responsible for any errors or omissions in this version of the manuscript or any version derived from it. The Version of Record is available online at https://iopscience.iop.org/article/10. 1088/1361-6560/ab8afc

Keywords: proton CT, small animal imaging, pre-clinical research, proton therapy, micromegas

Submitted to: Phys. Med. Biol.

\section{Introduction}

Pre-clinical in vivo irradiation studies with small animals are of paramount importance for translational cancer research in order to bridge the gap between in vitro cell experiments and clinical realization (Tillner et al. 2014). Various research platforms for photon-based pre-clinical irradiation studies have been developed and commercialized during the last decade (Verhaegen et al. 2011, Verhaegen et al. 2018). However, those systems are typically not applicable for nowadays emerging proton beam irradiations, except for rare cases (Ford et al. 2017).

The main benefit of proton irradiation stems from the characteristic rise in energy deposition towards the end of the proton beam range, the so-called Bragg peak. However, the resulting steep dose gradient also bears the risk of inflicting severe damage to healthy tissue if the proton beam range is not precisely known. A major source of range uncertainties is the imaging data used for treatment planning, typically relying on X-ray CTs expressed in the Hounsfield unit (HU) scale. For clinical cases, the uncertainty inherent in the typically employed stoichiometric conversion of $\mathrm{HU}$ values into the required ion relative (to water) stopping power (RSP) is around 3\% (Yang et al. 2012). Furthermore, for pre-clinical research with mice it is common practice to employ human reference data for dose calculation (Schyns et al. 2019), due to the limited availability of detailed information on murine tissue compositions in the literature. This potentially introduces additional range uncertainties, since the elemental composition of murine and human tissues can substantially differ.

Proton computed tomography (pCT) represents a promising replacement for Xray $\mathrm{CT}$ in proton therapy treatment planning since it allows to directly reconstruct 3D RSP distributions. Thus, aforementioned uncertainties originating from the HURSP conversion can be in principle completely eliminated. pCT has experimentally demonstrated excellent RSP accuracy (Dedes et al. 2019) and simulation studies have shown superior range calculation accuracy compared to X-ray CT (Meyer et al. 2019). Moreover, pCT images do not exhibit metal artifacts (Oancea et al. 2018) and can be obtained at extremely low dose exposure (Schulte et al. 2005), in particular 
if complementary fluence modulation is employed (Dedes et al. 2017, Dickmann et al. 2019).

Originally proposed in the 1960s, imaging with ion beams has experienced a revived interest by various research groups worldwide in the last 20 years (Poludniowski et al. 2015, Johnson 2018). While the main focus is on proton imaging, also heavier ions like helium or carbon ions have demonstrated promising results due to their reduced multiple Coulomb scattering (Volz et al. 2017, Gehrke et al. 2018, Magallanes et al. 2019). State-of-the-art in pCT imaging is the so-called single-particle tracking technique, which relies on the measurement of individual protons. To this end, pCT systems consist of two main components: a residual range/energy detector and a tracking system. The latter typically employs two pairs of $2 \mathrm{D}$ position-sensitive tracking detectors before and behind the imaged object, providing information on proton position and direction in order to estimate the individual trajectories throughout the object. The residual energy or range of every transmitted proton is measured with a calorimeter or range telescope (or hybrid system) placed downstream. This provides the energy lost by a proton inside the object, which is commonly expressed in terms of water-equivalent path length (WEPL). Nevertheless, despite tremendous advances in detector technology and reconstruction algorithms, pCT has not yet entered the clinical environment and is also barely investigated for pre-clinical imaging.

The SIRMIO project (Parodi et al. 2019) aims to provide a link between proton beam therapy and pre-clinical research by developing a dedicated platform for small animal proton irradiation. In order to achieve a high-precision irradiation, the platform will be equipped with a dedicated pCT system tailored to small animal imaging.

In this work, we present a Monte Carlo (MC) study on the feasibility and

performance of the aforementioned pCT system. For the envisaged construction of a first prototype, a geometrical design optimization of the detector components was conducted. This is necessary in order to enable an operation close to the intrinsic physical performance limits. Furthermore, we evaluated the overall imaging capabilities and provide the first demonstration of the potential of $\mathrm{pCT}$ for treatment planning in pre-clinical scenarios.

\section{Materials and methods}

\subsection{Principle of proton computed tomography}

For proton energies relevant in pCT, i.e., sufficiently high to fully traverse the imaged object, the mean energy loss $\mathrm{d} E$ in matter per unit path length $\mathrm{d} l$ is well described by the Bethe-Bloch (Bethe 1930) equation

$$
-\frac{\mathrm{d} E(E, \vec{r})}{\mathrm{d} l}=S(E(\vec{r}) I(\vec{r}))
$$

where $S$ is called (electronic) stopping power and $E$ and $I$ are the proton energy and material mean excitation energy at position $\vec{r}$, respectively. Within the context of pCT, 
equation 1 can be rewritten as the integral RSP along a proton trajectory $L$

$$
\int_{L} \operatorname{RSP}(\vec{r}) \mathrm{d} l=-\int_{E_{\text {in }}}^{E_{\text {out }}} \frac{\mathrm{d} E}{S_{w}\left(I_{w}, E\right)} \equiv \mathrm{WEPL},
$$

where $S_{w}$ and $I_{w}$ are the stopping power and mean excitation energy of water, respectively. The inverse problem of equation 2 represents the mathematical foundation of pCT since it enables a direct reconstruction of the 3D RSP distribution inside the imaged object. Within this work, we employed the iterative ordered-subset simultaneous algebraic reconstruction technique (Wang \& Jiang 2004) coupled to a total variation superiorization scheme (Penfold et al. 2010) for tomographic image reconstruction.

In order to minimize undesired effects of nuclear reactions and large-angle scattering, the statistical $3 \sigma$ event filter (Schulte et al. 2008) was implemented for parallel-ray projection bins. The air gap surrounding the imaged object was accounted for by computing a convex hull contour from an initial filtered back projection reconstruction. Subsequently, a straight line path through the object was assumed for every proton.

\subsection{Detector system and design optimization}

The proposed pCT system is based on planar gaseous particle detectors of the Micromegas (MICRO-MEsh GASeous) type (Giomataris et al. 1996). Micromegas are asymmetric parallel plate avalanche chambers with a several millimeter wide drift region and a sub-millimeter amplification region, separated by a thin conductive woven stainless steel micro-mesh held on supportive pillars. The Townsend electrons are detected at the charge-sensitive readout strips, which are individually connected to high-voltage and capacitively coupled to the electronics, so-called 'floating strips' (Bortfeldt 2014). This design massively reduces performance deterioration due to nondestructive discharges. Thus, Micromegas allow to reliably detect individual particles at fluxes of up to $60 \mathrm{MHz} / \mathrm{cm}^{2}$ and enable excellent spatial and good multi-hit resolution (Bortfeldt et al. 2016).

Within this work, Micromegas detectors in two different functionalities are investigated. The tracking detectors each consist of a doublet of novel 2D position sensitive Micromegas tracking detectors (Bortfeldt et al. 2017, Klitzner 2019). Here, the beam enters perpendicularly to the readout structure, hence, directly providing $2 \mathrm{D}$ position information. In the conventional approach, the anode is formed by $33 \mu \mathrm{m}$ thick photo-lithographically etched copper strips, which are individually connected to high-voltage. In order to obtain $2 \mathrm{D}$ position information, signals are registered via two additional layers of copper strips (one perpendicular and one parallel with respect to the floating strips). To reduce the material budget, two modifications of this configuration are proposed. First, the parallel position information can be directly retrieved by decoupling signals capacitatively from the floating strips with capacitors outside the

active area, which allows to remove the last strip layer. Additionally, the copper strips can be replaced by $9 \mu \mathrm{m}$ thick aluminum strips glued onto insulating Kapton. 
The residual range detector functions as a range telescope and is based on a timeprojection-chamber (TPC) (Marx \& Nygren 1978) with Micromegas readout structure and integrated Mylar field-shaping absorbers placed parallel to the electric drift field. In order to obtain the best compromise between WEPL resolution and detector complexity, different absorber thicknesses $\mathrm{d}=\{250 ; 500 ; 750 ; 1000\} \mu \mathrm{m}$ were investigated. A proton track is retrieved from the position information, which is directly measured in horizontal direction by the readout pads and indirectly inferred in vertical direction from the measured electron drift time. The residual proton range, i.e., the TPC stage in beam direction detecting the last energy deposition, can be calibrated to WEPL as explained later on. A schematic of the complete pCT system is shown in figure 1 .

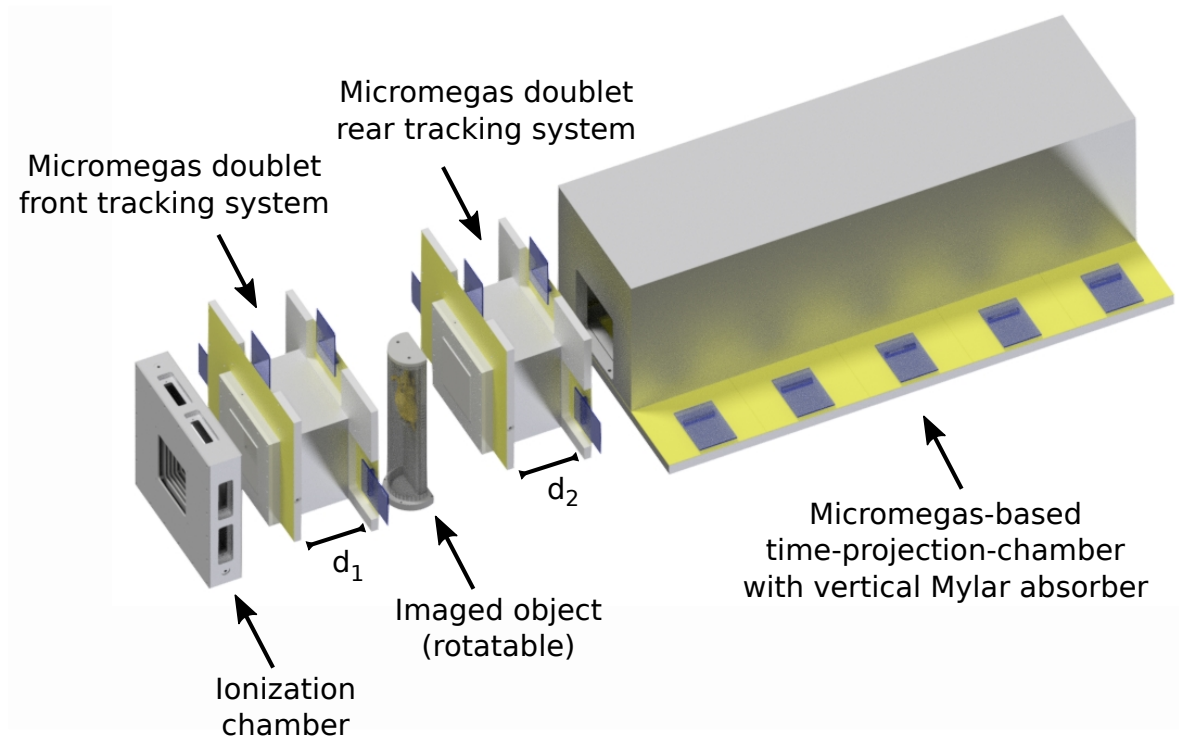

Figure 1: Schematic view of the SIRMIO pCT system components, including the precision dual strip ionization chamber for proton beam monitoring, both during imaging and treatment. The tracking systems located on both sides of the imaged object each consist of a doublet of $2 \mathrm{D}$ planar Micromegas detectors (separated by a distance $d_{1}$ and $d_{2}$ respectively). For measuring the proton residual range, a Micromegas-based TPC with vertical Mylar absorber plates. For improved visibility, the spacing of the components is exaggerated.

\subsection{Monte Carlo simulation framework}

All simulations were performed using the FLUKA (Ferrari et al. 2005, Böhlen et al. 2014) MC code (version 2011.2x.6 using HADROTHE defaults). The experimental beam characteristics of a ProBeam ${ }^{\circledR}$ beam line (Varian Medical Systems, Inc., Palo Alto, $C A, U S A$ ) were implemented for scanned proton beams of $75 \mathrm{MeV}$ nominal energy (i.e., a range in water of around $46.2 \mathrm{~mm}$ ), using the approach of Würl et al. (2016). 
The simulation model of the Micromegas tracking detector system explicitly considered the used materials and geometries, except for neglecting the fill factor of the micro-mesh by assuming a homogeneous iron layer of $18 \mu \mathrm{m}$ thickness. In order to incorporate the expected spatial resolution of the position-sensitive detectors, an uncertainty (randomly sampled from a normal distribution with conservatively chosen $\sigma=80 \mu \mathrm{m}$ (Bortfeldt et al. 2016)) was added to the obtained lateral position values. The range telescope was modeled as alternating structures of $5 \mathrm{~mm}$ wide gas-filled regions and homogeneous Mylar absorbers. For each proton, the integrated energy deposition within each gas layer in between two consecutive absorbers was scored through dedicated FLUKA user routines; however, solely the residual range is later used (i.e., the available position information at each layer is not employed). Additionally, a model of the parallel plate ionization chamber, foreseen to be permanently installed in the SIRMIO system for beam monitoring, was included in the simulation (upstream of the front tracker). All gaseous detectors contained an 80:20 vol. \% mixture of $\mathrm{Ne}: \mathrm{CF}_{4}$ at atmospheric pressure and the electric field was neglected.

The in silico phantom geometries described later on were modeled directly within the MC simulation according to the their physical dimensions and the manufacturer material composition specification. X-ray CT data of a mouse (isotropic voxel size of $0.2 \mathrm{~mm}$ ) in DICOM format was imported into FLUKA using the standard HU segmentation into 24 different materials of defined elemental composition and nominal mean density (Parodi et al. 2007). Electromagnetic processes were re-scaled to match a bijective clinical-like HU-RSP conversion curve. The knowledge of the exact (i.e., error-free) conversion curve allows to directly access the RSP ground truth reference.

\subsection{System performance characterization and physical limitations}

The achievable RSP accuracy depends on the detector WEPL resolution, which itself is intrinsically limited by the total range straggling. For a residual range measurement, this uncertainty is composed of the initial energy spread of the particle beam and the statistical energy loss fluctuations during the proton slowing-down process. The latter corresponds to around $1.1 \%$ of the mean range (Bashkirov et al. 2016). Based on the analytical relationship between initial energy $E_{0}$ and range $R$ according to Bortfeld (1997) $\left(R=\alpha E_{0}^{p}\right.$ with $\alpha=0.0022 \mathrm{~cm} / \mathrm{MeV}^{\mathrm{p}}$ and $\left.p=1.77\right)$, the additional range straggling due to the initial Gaussian momentum spread can be derived through Gaussian error propagation. For the simulated proton beams of $75 \mathrm{MeV}$ nominal energy with $1 \%$ momentum spread the total range straggling equals to $0.83 \mathrm{~mm}$.

The WEPL resolution was quantified according to the procedure of Bashkirov et al. (2016) by evaluating the detector response for different (water) absorber thicknesses. In order to quantitatively evaluate the achievable RSP accuracy, the pCT acquisition of a cylindrical water phantom $(\varnothing 30 \mathrm{~mm})$ with five tissue-equivalent insert rods $\varnothing$ $6 \mathrm{~mm}$ ) was simulated (cf. figure 5a). The insert materials were muscle, liver, adipose, trabecular bone200 and bone400 (CIRS, Inc., Norfolk, VA, USA), and details on the 
elemental composition can be found in Hudobivnik et al. (2016).

The precision of the estimated ion trajectories within the imaged object strongly influences the spatial resolution of the reconstructed image. In addition to the intrinsic inaccuracy of the selected trajectory model, the path estimation accuracy depends on four extrinsic factors, two related to the tracker configuration and two to its characteristics (Bopp et al. 2014). The latter are given by: (1) the single layer spatial resolution of the position-sensitive tracking detectors and (2) the material budget of the innermost (i.e., facing the imaged object) layer of the Micromegas doublet, which determines the amount of scattering a particle undergoes without the possibility to measure it. The limitations due to the tracker configuration can be characterized by: (3) the spacing between the individual sub-systems/layers within one Micromegas doublet (i.e., $d_{1}$ and $d_{2}$ in figure 1) and (4) the distance between object and tracker module, causing an amplification of uncertainties in position and direction estimation.

The path estimation accuracy was investigated in simulations of a $2 \mathrm{~cm}$ thick water phantom. The root mean square (RMS) deviation between the actual MC proton trajectories and the estimated paths was calculated for $10^{5}$ proton histories at 41 equally spaced points along the paths. Moreover, the pCT image spatial resolution was quantified using a slanted-edge phantom (i.e., a water phantom with a square insert of $8 \mathrm{~mm}$ side length and $2^{\circ}$ inclination as shown in figure 3a) (Mori \& Machida 2009). Due to the symmetry of the phantom, an average composite edge-spread function was obtained by combining the oversampled profiles from each side of the insert. The spatial resolution is quantified in terms of the $10 \%$ modulation transfer function.

\subsection{Small animal treatment planning}

To investigate the suitability for treatment planning of the reconstructed pCT images, a research version of the MC-based proton treatment planning system $\mathrm{\mu}$-RayStation (RaySearch Laboratories, Stockholm, Sweden) was used. Prior to the data import the images were back-converted into HUs using the inverse HU-RSP conversion curve implemented in the MC simulations. Due to the imposed bijectivity of that function, no uncertainty or ambiguity is introduced by this procedure (Meyer et al. 2019). Treatment plans were optimized on the ground truth using the beam properties of the SIRMIO beam line, obtained from validated MC simulations (Parodi et al. 2019,

Kurichiyanil et al. 2019), and subsequently recalculated on the pCT images. The dosimetric agreement was quantified in terms of (water-equivalent) beam's-eye view range differences (Meyer et al. 2019) for a hypothetical brain and lung tumor treatment.

\section{Results}

\subsection{Tracking detector performance and optimization}

The Micromegas spatial resolution is i.a. determined by the pitch of the readout strips and is (conservatively) assumed to yield $80 \mu \mathrm{m}$. Moreover, the distance between detector 
front face and isocenter must allow sufficient space for the object being imaged and is consequently fixed at $4 \mathrm{~cm}$. Since the intrinsic path model uncertainty is rather low (i.e., maximum RMS error of $0.07 \mathrm{~mm}$ ) due to the limited amount of multiple Coulomb scattering inside of $2 \mathrm{~cm}$ water, only two points for optimization remain: the spacing between the individual Micromegas planes in a doublet and their material budget.

The total RMS path estimation deviation for the three different readout structure configurations is shown in figure $2 \mathrm{a}$ as a function of depth inside the water phantom. The average (i.e., for the entire path within the object) RMS path deviation for the configuration with aluminum strips was around $0.29 \mathrm{~mm}$, and thus $0.07 \mathrm{~mm}$ and $0.10 \mathrm{~mm}$ lower than for the copper-based designs with two and three layers of strips in the readout structure, respectively.

The distance between the inner and outer detector of each Micromegas doublet influences the precision of the track angle measurement. Hence, the distance between the layers in the upstream and downstream tracker doublets was varied from $1 \mathrm{~cm}$ to $10 \mathrm{~cm}$ in $0.5 \mathrm{~cm}$ increments independent of one another. For the detector system with aluminum strip readout the resulting surface plot of the average RMS path estimation error is shown in figure $2 \mathrm{~b}$. Increasing upstream and downstream tracker spacing to more than around $7 \mathrm{~cm}$ did not considerably improve the path accuracy. This configuration yielded an average RMS path deviation of $0.18 \mathrm{~mm}$. As a cautious choice to avoid potential spatial resolution degradation in the image, $10 \mathrm{~cm}$ spacing of the tracker subsystems will be used for front and rear tracker hereafter.

For the aluminum-based design, the reconstructed pCT image of the slanted-edge phantom and the regions used for the construction of the oversampled edge profile can be seen in figure $3 \mathrm{a}$. The resulting modulation transfer functions for the different tracker configurations are shown in figure $3 \mathrm{~b}$. The obtained spatial resolution was $1.9 \mathrm{~mm}^{-1}$, $2.2 \mathrm{~mm}^{-1}$ and $2.8 \mathrm{~mm}^{-1}$ for the copper-based designs with three and two layers, and the aluminum strips configuration, respectively. The results are close to the performance of an ideal tracker system (obtained by using the MC information on the particles' position and direction at the entrance/exit of the tracking modules), which enabled a spatial resolution of $3.4 \mathrm{~mm}^{-1}$.

\subsection{Range telescope performance and optimization}

The range-to-WEPL calibration plots are shown in figure 4a. Based on the established linear calibration function, the standard deviation of the detector response can be translated into WEPL standard deviation by using Gaussian error propagation. The resulting WEPL resolutions as a function of WEPL being imaged are shown in figure 4b. As expected, the value generally improves towards thinner absorbers and is mostly constant except for statistical fluctuations. For increasing absorber thicknesses a systematically deteriorated performance toward lower WEPL values can be observed.

An exemplarily reconstructed pCT slice of the cylindrical insert phantom can be seen in figure 5a. The corresponding RSP accuracy for the insert materials achieved 


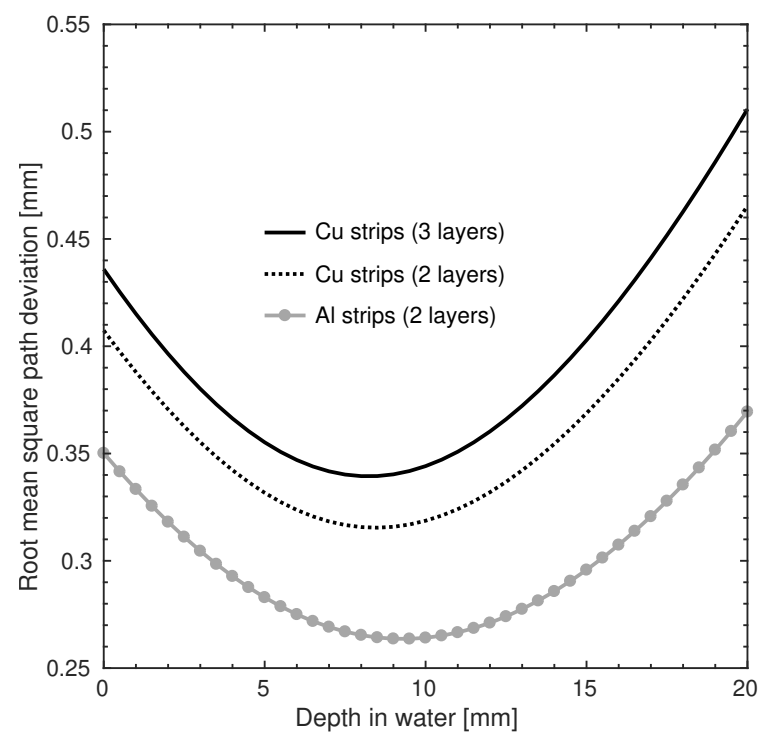

(a)

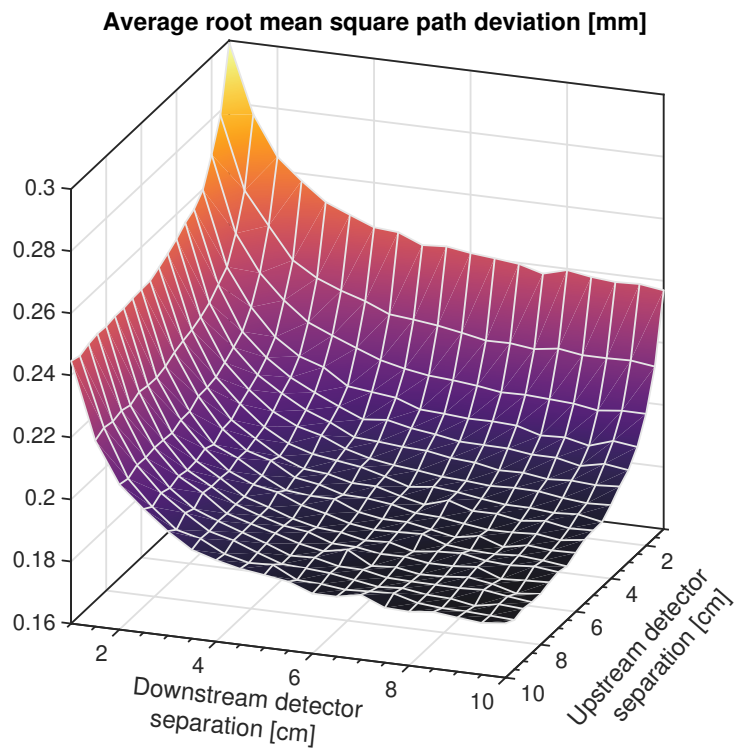

(b)

Figure 2: (a) Comparison of the total RMS path estimation error for the three different readout structure designs. (b) Surface plot of the average (i.e., for the entire path within the object) RMS path estimation error for the aluminum-based readout structure configuration as a function of upstream and downstream detector separation.

for different absorber thicknesses is displayed as bar plot in figure 5b, reflecting the improved WEPL resolution for thinner absorber slabs. Overall, sub-1\% RSP accuracy was demonstrated; however, results for $1000 \mu \mathrm{m}$ absorber thickness resulted in image artifacts of slightly distorted insert geometries. The mean RSP accuracy was $0.53 \%$, $0.27 \%, 0.24 \%$ and $0.22 \%$ for the designs with a granularity of $1000 \mu \mathrm{m}, 750 \mu \mathrm{m}, 500 \mu \mathrm{m}$ and $250 \mu \mathrm{m}$ Mylar, respectively.

\subsection{Imaging of pre-clinical data sets}

Figure 6a shows the reconstructed pCT images of a mouse head for the optimized detector system (i.e., aluminum-based tracking detector layers, spaced $10 \mathrm{~cm}$, and TPC with $500 \mu \mathrm{m}$ thick Mylar absorbers) along with the ground truth and the result for an ideal detector. The images were obtained for a dose exposure of (93 \pm 5 ) mGy. While the images clearly resemble the reference anatomy at a low noise level, blurring due to the limited spatial resolution is immanent. The corresponding RSP distributions are displayed as joint histogram in figure $6 \mathrm{~b}$ for the realistic detector model. The mean RSP for bone-like materials $(\mathrm{HU}>1200)$ and soft tissue-tissue $(-500<\mathrm{HU}<0)$ are underestimated by up to 0.15 . 


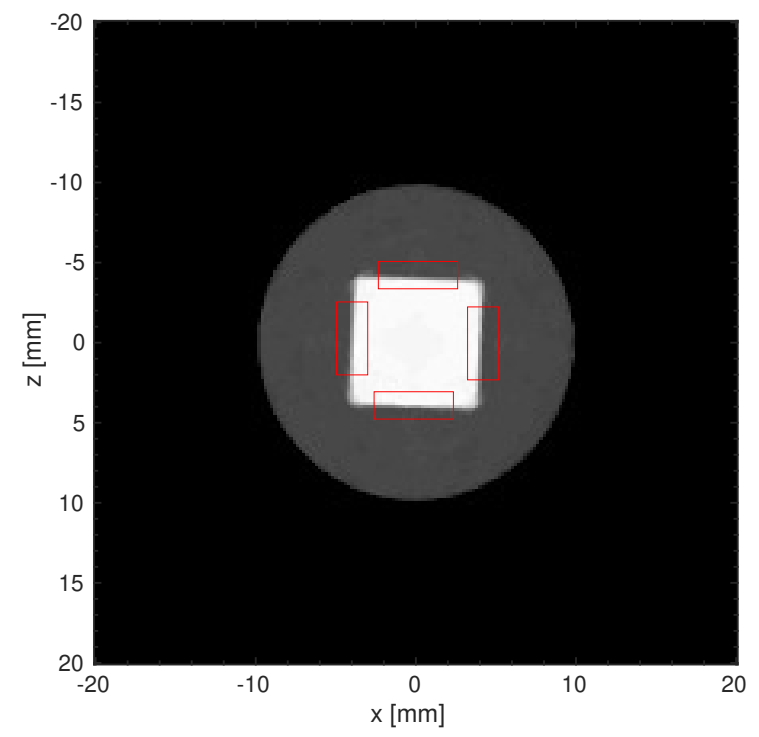

(a)

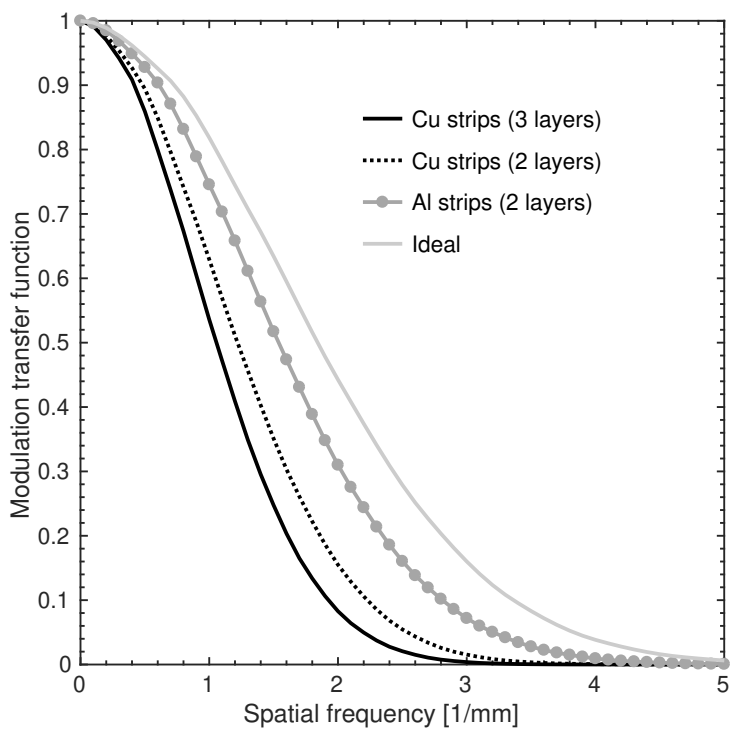

(b)

Figure 3: (a) Reconstructed pCT image of the slanted-edge phantom using an optimized aluminum-based tracker system. The indicated rectangles are the regions used for the construction of the oversampled edge profile and the viewing window is set between RSP values of 0.7 (black) and 1.7 (white). (b) Comparison of the corresponding modulation transfer functions for the different tracker configurations along with an ideal system.

\subsection{Treatment planning accuracy}

Figure 7 exemplary shows the optimized single field uniform dose distributions along with the results for plan recalculations on the reconstructed pCT images. In both cases, pCT enabled sub-millimeter accuracy with an average $( \pm 1 \sigma)$ relative proton-beam range error of $(-0.02 \pm 1.42) \%$ and $(+0.87 \pm 0.98) \%$ for the lung and brain case, respectively. The corresponding absolute water-equivalent range differences are $(-0.01 \pm 0.20) \mathrm{mm}$ and $(+0.09 \pm 0.10) \mathrm{mm}$.

\section{Discussion}

\subsection{Detector optimization}

Both proposed modifications of the design of previously existing tracking detectors resulted in a substantial performance benefit. Moving the layer of parallel readout strips out of the active area reduces the total material budget by around 33\%. The aluminumbased design exhibits 3.7 times lower overall metal thickness (from $33 \mu \mathrm{m}$ down to $9 \mu \mathrm{m}$ ) with about six times larger radiation length compared to copper $(8.897 \mathrm{~cm}$ and $1.436 \mathrm{~cm}$ respectively). These changes will substantially reduce multiple Coulomb scattering in the tracking detectors, which scales with the square-root of the material budget. Hence, 


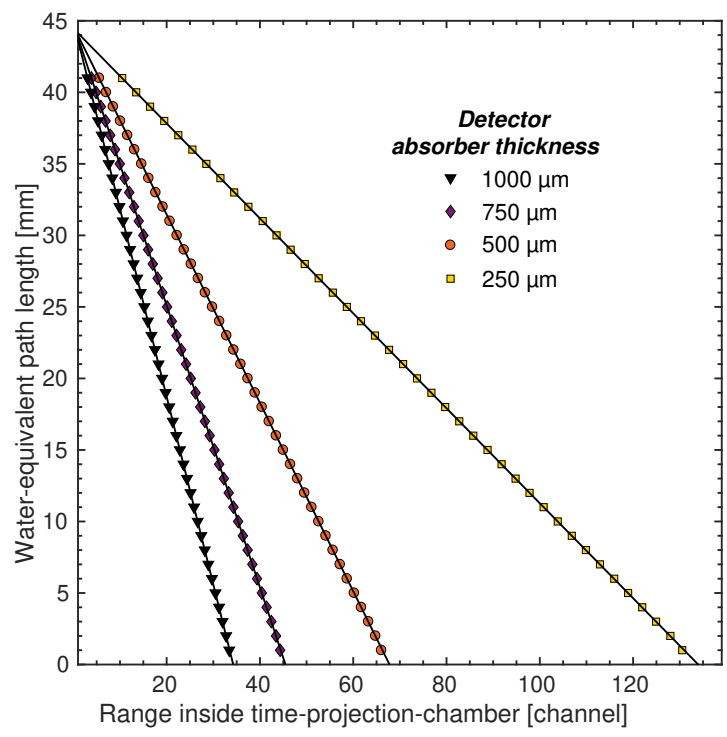

(a)

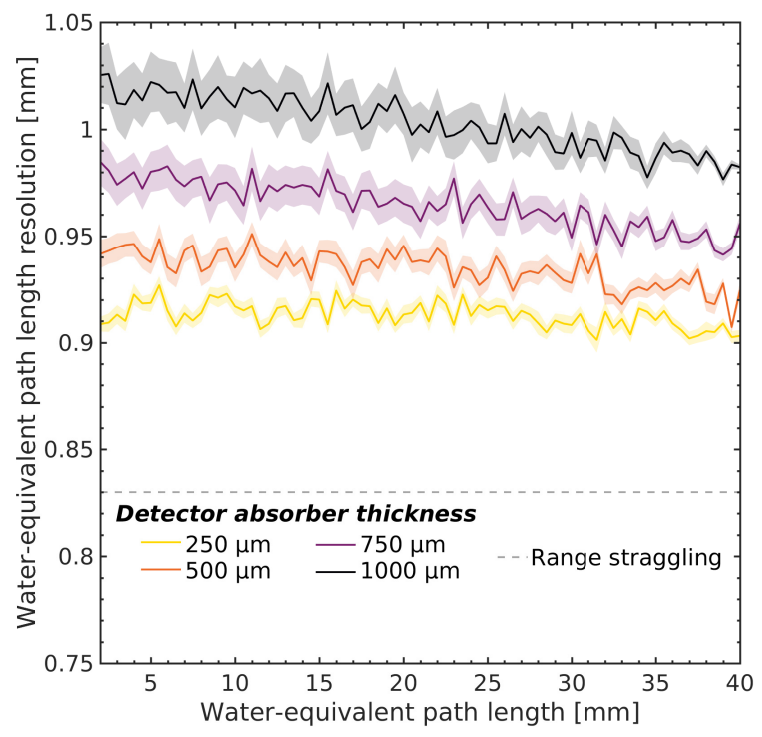

(b)

Figure 4: (a) Simulated calibration plots for the different TPC-based range telescope configurations: WEPL value versus range inside the detector obtained as mean value of the Gaussian fit. The black lines are linear fits used to extract the calibration functions. (b) WEPL resolution plots for the different TPC-based range telescope configurations as a function of WEPL being imaged: the intrinsic range straggling limit is indicated by the dashed gray line and the shaded area represents the $95 \%$ confidence interval obtained from the Gaussian fits.

these changes limit the amount of scattering a particle undergoes without the possibility to measure it. The path accuracy increases towards the object center (cf. figure 2a) since the information from both trackers is combined. In contrast, the intrinsic path model inaccuracy for relevant objects due to the stochastic nature of multiple Coulomb scattering, which is largest slightly downstream the center of the object, is rather small. For the same reason, the benefit of sophisticated proton path estimation models like the cubic spline path (Collins-Fekete et al. 2015) or most likely path (Schulte et al. 2008) over the computationally efficient straight line path is limited. In addition, the finite angular precision of the detector system is causing increased inaccuracies for those models exploiting the measured proton direction, in particular at the entrance and exit of the object. This can even lead to a superior performance of the straight line path for certain objects.

While the ideal detector layer spacing for the presented irradiation scenario was above $7 \mathrm{~cm}$, the value for the separation of the upstream tracker planes will generally depend on the absolute angular value (i.e., the divergence of the incident proton beam) since the angular uncertainty decreases with increasing absolute angular value. Furthermore, the optimum downstream value will vary according to the proton residual 


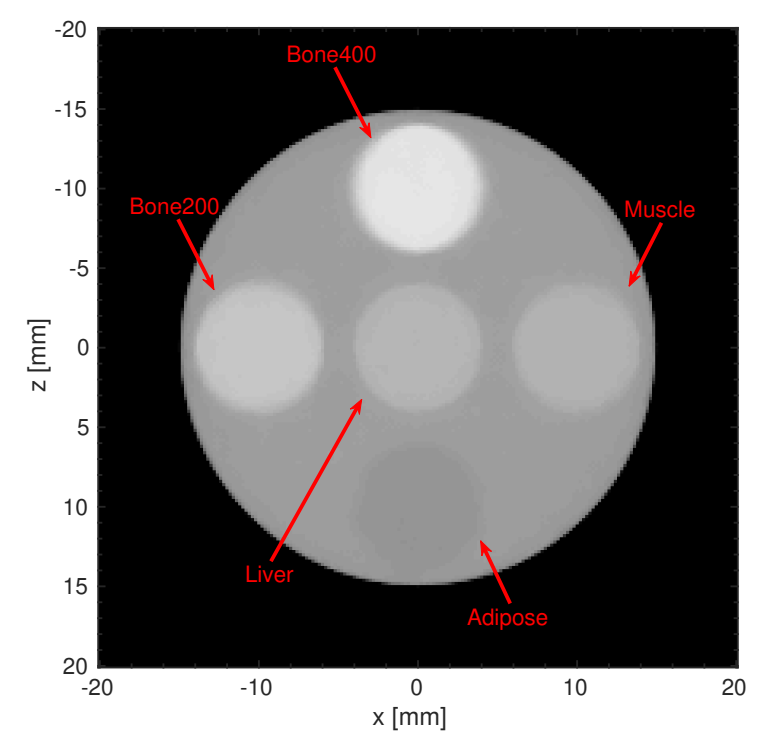

(a)

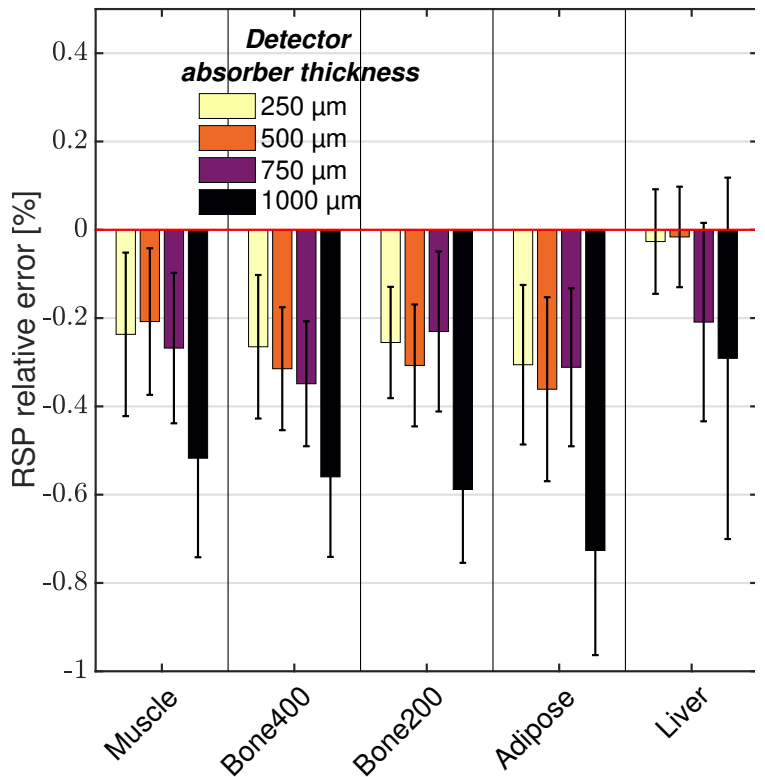

(b)

Figure 5: (a) Reconstructed pCT image of the cylindrical insert phantom obtained for an absorber thickness of $500 \mu \mathrm{m}$. The viewing window is set between RSP values of 0.7 and 1.7. (b) RSP relative error distributions for different absorber thicknesses displayed as bar plot. The bars correspond to the mean relative error values and whiskers represent the $\pm 1 \sigma$ intervals.

energy (i.e., shape and water-equivalent thickness of the object) (Penfold et al. 2011). This justifies the previous choice of $10 \mathrm{~cm}$ despite the reduced detector compactness.

Concerning the TPC, an absorber thickness between $500 \mu \mathrm{m}$ and $750 \mu \mathrm{m}$ represents the best trade-off between achievable WEPL/RSP resolution and detector complexity, since a larger quantity of thinner absorbers (and readout channels) would be required to enable the same dynamic WEPL range. The slightly degraded WEPL resolution toward smaller WEPL values (cf. figure 4b) might be a consequence of the large residual range within the TPC of the corresponding protons. Scattering and the initial particle direction could accumulate to a detectable difference between the CSDA range within the detector and the actually measured projected range in beam direction. This effect can potentially be compensated by applying an advanced residual range calibration. Future improvements could also encompass a more sophisticated event filtering as proposed by Volz et al. (2019) in conjunction with embedding the available TPC proton trajectory information. 


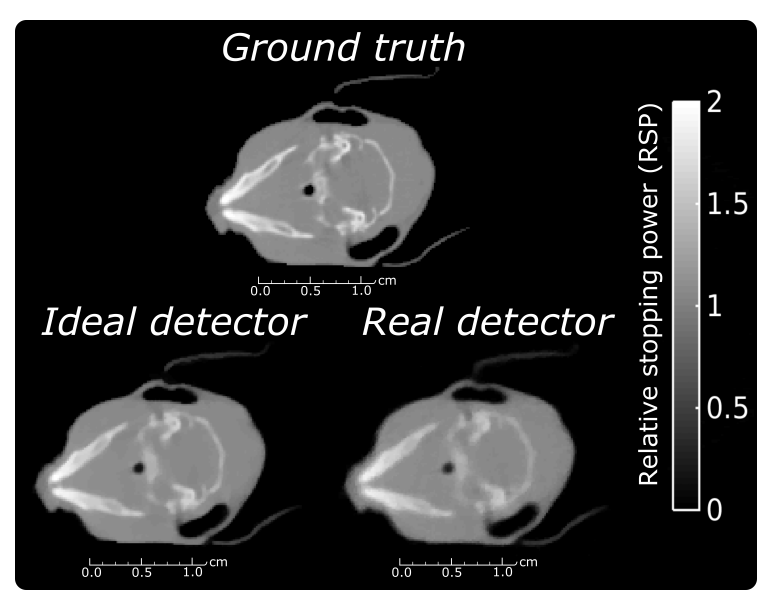

(a)

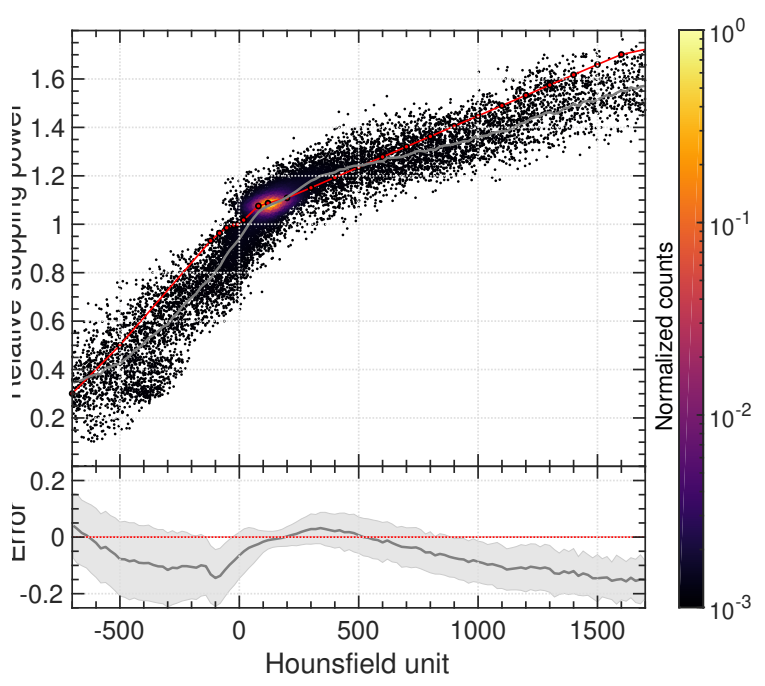

(b)

Figure 6: (a) Exemplary reconstructed pCT images for a mouse head obtained for an aluminum-based tracker and TPC with $500 \mu \mathrm{m}$ thick absorber foils along with the ground truth. (b) Corresponding joint histogram of the reconstructed RSP and the original HU values. The red line indicates the ground truth conversion curve being used to import the initial X-ray CT data into the MC simulation. The curve below shows the corresponding mean RSP error $( \pm 1 \sigma)$.

\subsection{Imaging performance}

The obtained spatial resolution of around $3 \mathrm{~mm}^{-1}$ for the optimized pCT system is comparable to the performance of cone beam CT systems commonly used in pre-clinical research (Song et al. 2010). However, the level of detail visible in the presented pCT reconstructions for realistic small animal data (cf. figure 6a) appears slightly inferior to the estimated spatial resolution based on the phantom study. One reason is the highly heterogeneous 3D anatomy, which creates additional path uncertainties compared to the rotationally symmetric homogeneous phantom case. Nevertheless, the observed sub- $0.5 \%$ RSP accuracy of pCT is expected to be substantially superior to X-ray CT imaging. The observed systematic minor RSP underestimation (cf. figure 5b) is also reflected in the reconstructions for pre-clinical data (cf. figure 6b). This once again promotes the investigation of more sophisticated TPC-range calibration methods.

For the study of pre-clinical small animal data, the mouse holder being developed in-house for the SIRMIO project was not included in the simulation. It consists of a rigid yet low material budget fixation system, heating and connections for air and anesthesia supply. In order to guarantee a sterile environment for immunodeficient mice, the chamber will be surrounded by a nearly cylindrical shell consisting of a $50 \mu \mathrm{m}$ thin Kapton foil (Parodi et al. 2019). To further reduce scattering for ultra-precise irradiation, the support bed will contain cutouts (sealed with a thin Kapton foil) at 

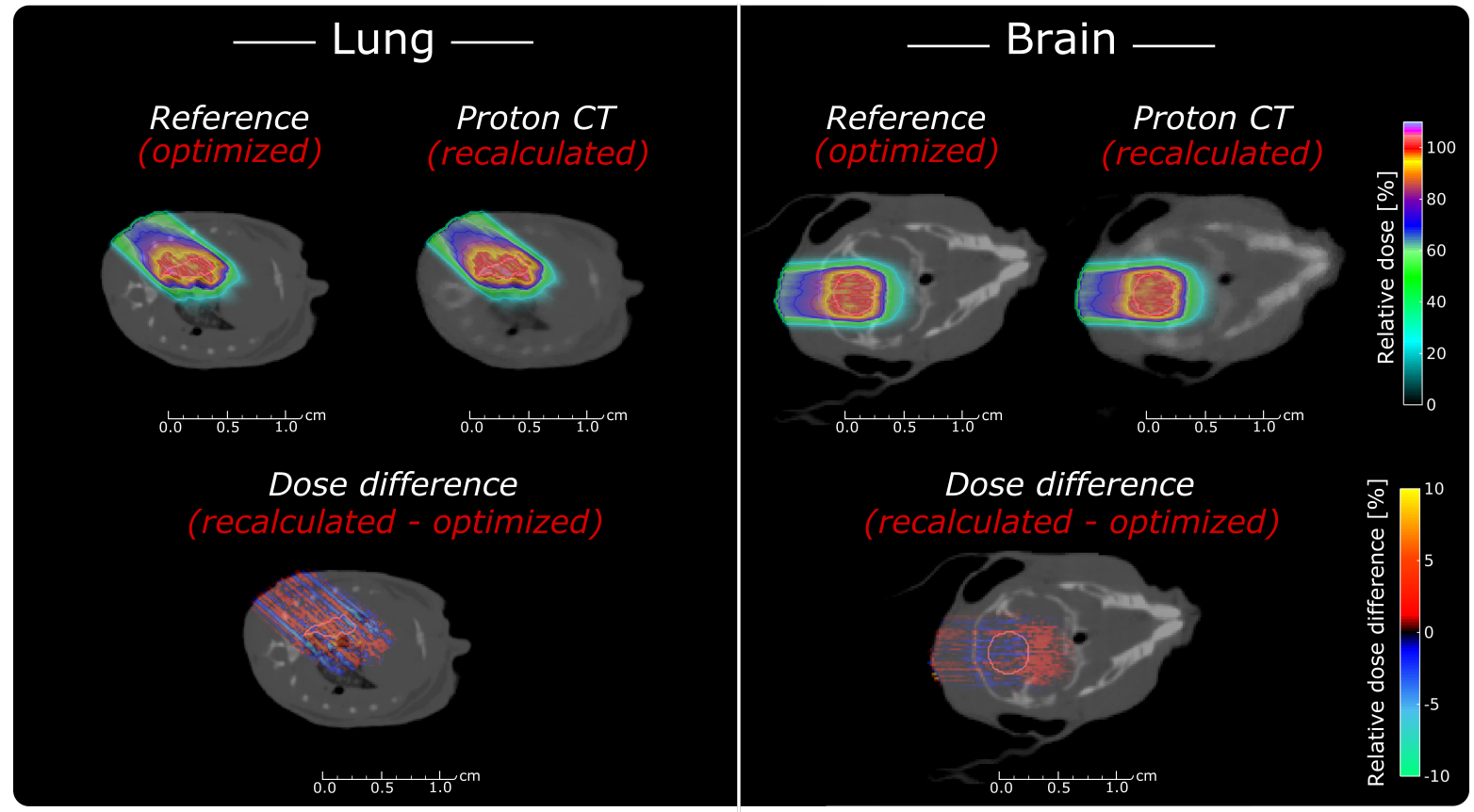

Figure 7: Treatment plans for a hypothetical lung (left) and brain (right) tumor case in a mouse model. Relative dose distributions (with respect to the prescribed target dose) are shown for the optimized reference plans and the recalculated ones (i.e., on the pCT images) along with the corresponding relative dose differences.

irradiation locations specific to the treated indiciation. In order to compensate the potential deterioration of the $\mathrm{pCT}$ spatial resolution due to the additional material the tracking system could be moved slightly closer towards the holder.

\subsection{Treatment planning accuracy}

The observed range inaccuracies result as combination of limited RSP accuracy and spatial resolution. For the rather homogenous brain case, the uncertainty is dominated by the slight RSP underestimation for soft tissue, which accumulates to the detected over-ranging. In contrast, the lung case is dominated by heterogeneities, resulting in a better mean accuracy but increased standard deviation. Considering that a stoichiometric HU-RSP conversion based on human reference tissues for small animal irradiation will result in range uncertainties above $3 \%, \mathrm{pCT}$ is able to provide improved treatment planning accuracy compared to conventional X-ray CT. Moreover, pre-clinical imaging commonly only employs cone beam CT systems, which are typically providing only limited image quality. The pCT system will enable imaging of mice directly in treatment position (including a correction of the proton beam position via the installed ionization chamber). 


\subsection{Potential limitations of an experimental realization}

The detector system simulated in this study provides accurate imaging capabilities, however, there are some potential limitations regarding the translation from in silico to experimental realization. First, the manufacturing and structuring process of the aluminum-based Micromegas is more sophisticated compared to the conventional copper design and requires certain adjustments. Nevertheless, the process has been completely mastered in-house and a first prototype has been already produced and sucessfully tested in $22 \mathrm{MeV}$ proton beams. No aging has been observed. A detailed evaluation of the actual position resolution, which is possibly even better than the assumed $80 \mu \mathrm{m}$ is ongoing. The functional principle of the proposed TPC configuration has been demonstrated with a first prototype (Lämmer 2019). A reliable electron extraction within the active region in between two consecutive Mylar plates is has been demonstrated for $6 \mathrm{~mm}$ absorber spacing using field-shaping foils.

\section{Conclusion}

The presented work is the first detailed investigation of the potential of pCT with a single-particle tracking system for small animal imaging. It provides a thorough MC-based optimization of the detector configuration and quantitatively evaluates the expected performance with respect to the requirements for pre-clinical research. Both components of the pCT system are currently under construction in-house and first prototypes have been successfully tested in proton beams, underlining the feasibility of the proposed system. While proton imaging is nowadays on the verge of becoming clinically available, the proposed pre-clinical system will contribute to an improved precision of small animal proton irradiations. This might help to provide a better understanding of the fundamental in vivo characteristics of cancer and normal tissue response to ion radiation exposure.

\section{Acknowledgments}

This work has been financially supported by the ERC Consolidator Grant SIRMIO (grant number 725539). JB. acknoledges support from the COFUND-FP-CERN-2014 program (grant number 665779). Dr. Chiara Gianoli is acknowledged for fruitful discussions. Furthermore, the authors thank Prof. Frank Verhaegen for providing the mouse CT data and Drs. Erik Traneus and Rasmus Nilsson for support with the treatment planning software.

\section{Disclosure statement}

The Medical Physics Department of the Ludwig-Maximilians-Universität München has a license and research collaboration agreement with RaySearch Laboratories AB (Stockholm, Sweden) for the $\mu$-RayStation proton therapy treatment planning system. 


\section{References}

Bashkirov V A, Schulte R W, Hurley R F, Johnson R P, Sadrozinski H F W, Zatserklyaniy A, Plautz T \& Giacometti V 2016 Med. Phys. 43(2), 664-674.

Bethe H 1930 Annalen der Physik 397, 325-400.

Böhlen T, Cerutti F, Chin M, Fassò A, Ferrari A, Ortega P, Mairani A, Sala P, Smirnov G \& Vlachoudis V 2014 Nuclear Data Sheets 120, 211-214.

Bopp C, Rescigno R, Rousseau M \& Brasse D 2014 Physics in Medicine \& Biology 59(23), N197-N210.

Bortfeld T 1997 Medical Physics 24(12), 2024-2033.

Bortfeldt J 2014 Development of Floating Strip Micromegas Detectors PhD thesis Ludwig-MaximiliansUniversität München.

Bortfeldt J, Bender M, Biebel O, Danger H, Flierl B, Hertenberger R, Lösel P, Moll S, Parodi K, Rinaldi I, Ruschke A \& Zibell A 2016 Nuclear and Particle Physics Proceedings 273, 1173-1179.

Bortfeldt J, Biebel O, Flierl B, Hertenberger R, Klitzner F, Lösel P, Magallanes L, Müller R, Parodi K, Schlüter T, Voss B \& Zibell A 2017 Nuclear Instruments and Methods in Physics Research Section A: Accelerators, Spectrometers, Detectors and Associated Equipment 845, 210-214.

Collins-Fekete C A, Doolan P, Dias M F, Beaulieu L \& Seco J 2015 Physics in Medicine 6 Biology 60(13), 5071.

Dedes G, Angelis L D, Rit S, Hansen D, Belka C, Bashkirov V, Johnson R P, Coutrakon G, Schubert K E, Schulte R W, Parodi K \& Landry G 2017 Physics in Medicine E Biology 62(15), 6026-6043.

Dedes G, Dickmann J, Niepel K B, Wesp P, Johnson R P, Pankuch M, Bashkirov V A, Rit S, Volz L, Schulte R W, Landry G \& Parodi K 2019 Physics in Medicine 83 Biology .

Dickmann J, Wesp P, Rädler M, Rit S, Pankuch M, Johnson R P, Bashkirov V, Schulte R W, Parodi K, Landry G \& Dedes G 2019 Physics in Medicine \& Biology 64(14), 145016.

Ferrari A, Sala P, Fassò A \& Ranft J 2005 FLUKA: a multi-particle transport code Technical report CERN-2005-10 (2005), INFN/TC 05/11, SLAC-R-773.

Ford E, Emery R, Huff D, Narayanan M, Schwartz J, Cao N, Meyer J, Rengan R, Zeng J, Sandison G, Laramore G \& Mayr N 2017 Physics in Medicine \& Biology 62(1), 43-58.

Gehrke T, Gallas R, Jäkel O \& Martišíková M 2018 Medical Physics 45(2), 817-829.

Giomataris Y, Rebourgeard P, Robert J \& Charpak G 1996 Nuclear Instruments and Methods in Physics Research Section A: Accelerators, Spectrometers, Detectors and Associated Equipment 376(1), 29-35.

Hudobivnik N, Schwarz F, Johnson T, Agolli L, Dedes G, Tessonnier T, Verhaegen F, Thieke C, Belka C, Sommer W H, Parodi K \& Landry G 2016 Medical Physics 43(1), 495-504.

Johnson R P 2018 Reports on Progress in Physics 81(1), 016701.

Klitzner F 2019 Research and Development of Two-Dimensional Floating Strip Micromegas Detectors $\mathrm{PhD}$ thesis Ludwig-Maximilians-Universität München.

Kurichiyanil N, Pinto M, Rösch T, Kundel S, Wuerl M, Englbrecht F, Schreiber J \& Parodi K 2019 Medical Physics 46(6), e425.

Lämmer P 2019 Studies on an ionization chamber and time projection chamber for beam position monitoring and ion transmission imaging Master's thesis Ludwig-Maximilians-Universität München.

Magallanes L, Meyer S, Gianoli C, Kopp B, Voss B, Jäkel O, Brons S, Gordon J \& Parodi K 2019 IEEE Transactions on Radiation and Plasma Medical Sciences .

Marx J N \& Nygren D R 1978 Phys. Today 31N10, 46-53.

Meyer S, Kamp F, Tessonnier T, Mairani A, C. B, Carlson D J, C. G \& K P 2019 Physics in Medicine E Biology 64(12), 125008.

Mori I \& Machida Y 2009 Radiological physics and technology 2(1), 22.

Oancea C, Shipulin K, Mytsin G, Gao M, Pankuch M, Coutrakon G, Ordonez C, Johnson R, Bashkirov V \& Schulte R 2018 Radiotherapy and Oncology 127, S470-S471.

Parodi K, Assmann W, Belka C, Bortfeldt J, Clevert D A, Dedes G, Kalunga R, Kundel S, Kurichiyanil 
N, Lämmer P, Lascaud J, Lauber K, Lovatti G, Meyer S, Nitta M, Pinto M, Safari M, Schnürle

K, Schreiber J, Thirolf P G, Wieser H P \& Würl M 2019 Acta Oncologica 10(58), 1470.

Parodi K, Ferrari A, Sommerer F \& Paganetti H 2007 Physics in Medicine \& Biology 52(12), 3369.

Penfold S N, Rosenfeld A B, Schulte R W \& Sadrozinksi H F W 2011 Radiation Measurements 46(12), 2069-2072.

Penfold S N, Schulte R W, Censor Y \& Rosenfeld A B 2010 Medical Physics 37(11), 5887-5895.

Poludniowski G, Allinson N \& Evans P 2015 The British journal of radiology 88(1053), 20150134.

Schulte R W, Bashkirov V, Loss Klock M C, Li T, Wroe A J, Evseev I, Williams D C \& Satogata T 2005 Medical Physics 32(4), 1035-1046.

Schulte R W, Penfold S N, Tafas J T \& Schubert K E 2008 Medical Physics 35(11), 4849-4856.

Schyns L E J R, Eekers D B P, van der Heyden B, Almeida I P, Vaniqui A \& Verhaegen F 2019 The British Journal of Radiology $\mathbf{9 2 ( 1 0 9 5 ) .}$

Song K H, Pidikiti R, Stojadinovic S, Speiser M, Seliounine S, Saha D \& Solberg T D 2010 Physics in Medicine and Biology 55(23), 7345-7362.

Tillner F, Thute P, Bütof R, Krause M \& Enghardt W 2014 Zeitschrift für Medizinische Physik 24(4), 335-351.

Verhaegen F, Dubois L, Gianolini S, Hill M A, Karger C P, Lauber K, Prise K M, Sarrut D, Thorwarth D, Vanhove C, Vojnovic B, Weersink R, Wilkens J J \& Georg D 2018 Radiotherapy and Oncology 126(3), 471-478.

Verhaegen F, Granton P \& Tryggestad E 2011 Physics in Medicine 83 Biology 56(12), R55-R83.

Volz L, Collins-Fekete C A, Piersimoni P, Johnson R, Bashkirov V, Schulte R \& Seco J 20173.

Volz L, Piersimoni P, Johnson R P, Bashkirov V A, Schulte R W \& Seco J 2019 Physics in Medicine G Biology 64(15), 15NT01.

Wang G \& Jiang M 2004 Journal of X-Ray Science and Technology 12, 169-177.

Würl M, Englbrecht F, Parodi K \& Hillbrand M 2016 Physics in Medicine \& Biology 61(2), 958.

Yang M, Zhu X R, Park P, Titt U, Mohan R, Virshup G, Clayton J \& Dong L 2012 Physics in Medicine E Biology 57(13), 4095-4115. 